signal selection in parameter estimation problems," Inform. Sci., vol. 12, pp. 61-91, 1977.

[5] - "Synthesis of linear stochastic signals in identification problems," Automatica, vol. 13, pp. 615-622, 1977.

[6] T. W. Kerlin, Frequency Response Testing in Nuclear Reactors. New York: Academic, 1974.

[7] M. D. Canon et al., Theory of Optimal Control and Mathematical Programming. New York: McGraw-Hill, 1970.

[8] B. R. Upadhyaya, "Synthesis of input signals in parameter estimation problems," Ph.D. dissertation, Univ. California, San Diego, 1975.

\section{Optimal Linear Stochastic Control for Systems with Multiplicative Noise}

\section{ARUNABHA BAGCHI AND TOM SCHILPEROORT}

Abstract-The stochastic control problem of a linear dynamical system with multiplicative noise and with incomplete and inaccurate observation, has been studied for quadratic performance criterion. A suboptimal solution, which is the best linear control based on the available observations, has been worked out when the observations are given only at discrete-time points.

\section{INTRODUCTION}

We obtain the best linear control law for a stochastic control problem with linear dynamics, but with multiplicative noise, based on observations at discrete-time points. We convert the problem into successive control problems where the control depends on the current observation only. Mclane [1] solved this type of problem using the matrix maximum principle of Athans [2]. This gives explicit control laws for our problem, which involves successive solutions of nonlinear boundary value problems.

\section{Problem Formulation}

We consider a dynamical system described by

$$
\begin{aligned}
& d X_{t}=A(t) X_{t} d t-B(t) U_{t} d t+D\left(t, X_{t}\right) d W_{1 t}+E(t) d W_{2 t} \\
& d Y_{t}=C(t) X_{t} d t+G\left(t, X_{t}\right) d W_{3 t}+F(t) d W_{4 t}, \quad 0<t<T .
\end{aligned}
$$

$X_{0}, Y_{0}$ are independent random vectors, $X_{t}$ is an $n$-dimensional state, $U_{t}$ is a $p$-dimensional control, $Y_{t}$ is an $m$-dimensional observation, and

$$
D\left(t, X_{t}\right)=\sum_{i=1}^{n} D_{i}(t) X_{i r}, G\left(t, X_{t}\right)=\sum_{i=1}^{n} G_{i}(t) X_{i t}
$$

with $X_{i t}$ being the $i$ th component of $X_{t}$ and $D_{i}(t)$ and $G_{i}(t)$ are appropriate dimensional matrices. $W_{i t}, i=1,2,3,4$, are independent Brownian motions of dimensions $d_{i}$, independent of $\boldsymbol{X}_{0}$ and $\boldsymbol{Y}_{\mathbf{0}}$. The matrices $A(t), B(t), C(t), E(t), F(t)$ have appropriate dimensions.

Let $t_{0}=0$ and $t_{k}=k T / N, k=1, \cdots, N$, and at these time points we observe the process $Y_{t}$. We can generalize to the case of nonequidistant $t_{k}{ }^{\prime} s$. For $t \in\left[t_{j-1}, t_{j}\right), j=1, \cdots, N$, let $Y^{j-1}=\operatorname{col}\left(Y_{t_{j-1}}, \cdots, Y_{0}\right)$. Let $\Phi_{k}=\left\{\phi_{j}:\left[t_{j-1}, t_{j}\right) X R^{m j} \rightarrow R^{P}\right.$ such that $\left.\phi_{j}(t, y)=\sum_{i=0}^{j=1} k_{i}(t) Y_{t_{i}}\right\}$. We denote by $\mathscr{Q}_{l j}$ the class of control $U_{j t}$ where

$$
U_{j t}=\phi_{j}\left(t, Y^{j-1}\right), \quad t \in\left[t_{j-1}, t_{j}\right), \phi_{j} \in \Phi_{k}^{j} .
$$

A control $U_{t}, t \in[0, T]$ is now admissible if

Manuscript received April 15, 1980.

A Bagchi is with the Department of Applied Mathematics, Twente University of Technology, Enschede, The Netherlands.

T. Schilperoort is with the Delft Hydraulics Laboratory, Laboratory "de Voorst," Emmeloord, The Netherlands.

$$
\begin{array}{ll}
\text { i) } U_{t}=U_{j t}, & t \in\left[t_{j-1}, t_{j}\right), j=1, \cdots, N \\
\text { ii) } U_{j t} \in Q_{t j}, & j=1, \cdots, N .
\end{array}
$$

Denote this class by $\mathcal{U}_{l a}$. We want to determine a control $U_{t}$ in this class that minimizes

$$
J(U)=E\left\{\int_{0}^{T}\left(X_{t}^{\prime} Q(t) X_{t}+U_{t}^{\prime} R(t) U_{\imath}\right) d t+X_{T}^{\prime} Q_{f} X_{T}\right\}
$$

with "prime" denoting transpose, where the matrices $Q(t)>0, Q_{f}>0$, and $R(t)>0$ a.e. $t$.

\section{Determination of the Optmal Lingar Control}

Let

$$
Z_{t}=\left(\begin{array}{c}
X_{t} \\
Y_{t}
\end{array}\right), \quad t \in[0, T]
$$

and $\bar{C}=\left(0_{m n} I_{m m}\right)$, where $0_{m n}$ is the $m \times n$ zero matrix and $I_{m m}$ is the $m \times m$ identity matrix, so that $Y_{t}=\bar{C} Z_{t}$. For $j=1, \cdots, N$, define stochastic processes

$$
Z_{i}^{j}=\left(\begin{array}{c}
Z_{t} \\
Z^{j-1}
\end{array}\right) \text {, with } Z^{j-1}=\operatorname{col}\left(Z_{t_{j-1}}, \cdots, Z_{0}\right), t \in\left[t_{j-1}, t_{j}\right) .
$$

Define, for $j=1, \cdots, N$, matrices of dimensions $p \times m j$ :

$$
K^{j}(t)=\left(K_{j-1}^{j}(t), \cdots, K_{b}^{j}(t)\right) .
$$

Let $\bar{C}^{j}$ be a $j \times(j+1)$ block $m \times(n+m)$ matrices with $\left(\bar{C}^{j}\right)_{k, k+1}=\bar{C}$ and $\left(\bar{C}^{j}\right)_{k l}=0$ for $l \neq k+1, k=1, \cdots, j ; l=1, \cdots, j+1$. A control $U_{j t} \in Q_{l j}$ can be expressed as $U_{j t}=K^{j}(t) \bar{C}^{j} Z_{i}^{j}$, so that, using this control, $Z_{i}^{j}$ is the solution of

$$
d Z_{i}^{j}=\left(A^{j}(t)-B^{j}(t) K^{j}(t) \bar{C}^{j}\right) Z_{i}^{j} d t+\sigma^{j}\left(t, Z_{i}^{j}\right) d W_{t}
$$

where

$$
\begin{gathered}
W_{t}=\operatorname{col}\left(W_{1 t}, W_{2 t}, W_{3 t}, W_{4 t}\right) \\
\sigma^{j}\left(t, Z_{t}^{j}\right)=\left(\sum_{i=1}^{n} D_{i}^{j}(t)\left(Z_{t}^{j}\right)_{i} E^{j}(t) \sum_{i=1}^{n} G_{j}^{j}(t)\left(Z_{t}^{j}\right)_{i} F^{j}(t)\right)
\end{gathered}
$$

and the matrices $A^{j}(t), B^{j}(t), D_{i}^{j}(t), E^{j}(t), G_{i}^{j}(t), F^{j}(t)$ have easily identifiable structure.

Define, for $t \in\left[t_{j-1}, t_{j}\right), j=1, \cdots, N$,

$$
L_{k}^{j}\left(t, z^{j}\right)=\left(z^{j}\right)^{\prime}\left[Q^{j}(t)+\left(\bar{C}^{j}\right)^{\prime} K^{j}(t)^{\prime} R(t) K^{j}(t) \bar{C}^{j}\right] z^{j}
$$

where $Q^{J}(t)=$ block $\operatorname{diag}(Q(t), 0,0)$ so that we have

$$
J=E\left\{\sum_{k=1}^{N} \int_{t_{k+1}}^{t_{k}} L_{K}^{k}\left(s, Z_{s}^{k}\right) d s+\left(Z_{T}^{N}\right)^{\prime} Q_{f}^{N} Z_{T}^{N}\right\}
$$

with $Q_{f}^{j}=$ block diag $\left(Q_{f}, 0,0\right)$.

Let

$$
J_{K}^{j}=E\left\{\sum_{k=j}^{N} \int_{t_{k-1}}^{t_{k}} L_{K}^{k}\left(s, Z_{s}^{k}\right) d s+\left(Z_{T}^{N}\right)^{\prime} Q_{f}^{N} Z_{T}^{N}\right\}
$$

and

$$
W_{K}^{k}=\int_{t_{k-1}}^{t_{k}} L_{K}^{k}\left(s, Z_{s}^{k}\right) d s
$$

Bellman's principle of optimality yields the following procedure for determining the optimal control sequence $U^{*}{ }_{1}, \cdots, U^{*}{ }_{N t}$. 
1) Find $U_{N t} \in \mathscr{Q}_{1 N}$ for which $J_{K}^{N}$ is a minimum. Use $J_{K}^{i *}$ to denote the minimum value of $J_{k}^{j}, j=1, \cdots, N$.

2) Find, successively, for $j=(N-1), \cdots, 1, U_{j t} \in Q_{1 j}$ for which

$$
E\left\{W_{k}^{j}+J_{K}^{(j+1)^{*}}\right\}
$$

is a minimum.

To carry out these minimizations, consider functions $V_{k}\left(t, z^{j}\right), j=$ $1, \cdots, N$, which are solutions of the backward equations

$$
\begin{aligned}
\frac{\partial V \dot{k}\left(t, z^{j}\right)}{\partial t}+\mathcal{E}_{K}\left(t, z^{j}\right) V \dot{k}\left(t, z^{j}\right)+L j\left(t, z^{j}\right)=0 & \\
\cdot\left(t, z^{j}\right) & \in\left[t_{j-1}, t_{j}\right) \times R^{(n+m)(j+1)}
\end{aligned}
$$

with the final conditions, for $j=N$,

$$
V_{K}^{N}\left(T, z^{N}\right)=\left(z^{N}\right)^{\prime} Q_{f}^{N} z^{N}
$$

and for $j=N-1, \cdots, 1$,

$$
V_{k}^{j}\left(t_{j}, z^{j}\right)=V_{K}^{j+1}\left(t_{j}, z^{j}, z_{j}\right)
$$

where the differential generator $\mathfrak{L}_{k}\left(t, z^{j}\right), z^{j} \in R^{(n+m)(j+1)}$ is defined by

$$
\begin{aligned}
\mathcal{E}_{K}\left(t, z^{j}\right)=\left(z^{j}\right)^{\prime}\left[A^{j}(t)-B^{j}(t) K^{j}(t) \bar{C}^{j}\right]^{\prime} \frac{\partial}{\partial z^{j}} & \\
+ & +\frac{1}{2} \operatorname{tr}\left[\sigma^{j}\left(t, z^{j}\right) \sigma^{j}\left(t, z^{j}\right)^{\prime} \frac{\partial^{2}}{\partial\left(z^{j}\right)^{2}}\right] .
\end{aligned}
$$

The system of equations (3.7), (3.8a), (3.8b) has a unique solution which follows from standard existence results in partial differential equations with slight modification [3]. The Itô differentiation rule [4] gives

$$
J_{k}=E\left\{V_{k}^{j}\left(t_{j-1}, Z_{t_{j-1}}^{j}\right)\right\}, \quad j=1, \cdots, N .
$$

We propose a solution of (3.7) in the form

$$
V_{K}^{j}\left(t, z^{j}\right)=\left(z^{j}\right)^{\prime} P_{K}^{j}(t) z^{j}+p_{K}^{j}(t)
$$

with $P_{X}^{j}(t) \in H_{(n+m)(j+1)}, j=1, \cdots, N$, where $H_{n}$ stands for the class of all $n \times n$ symmetric matrices.

Substituting (3.11) in (3.7), we get for $j=1, \cdots, N$,

$\dot{P}_{K}^{j}+P_{K}^{j}\left(A^{j}-B^{j} K^{j} \bar{C}^{j}\right)+\left(A^{j}-B^{j} K^{j} \bar{C}^{j}\right)^{\prime} P_{K}^{j}+Q^{j}$

$$
+\Delta^{j}\left(t, P_{k}^{j}\right)+\Gamma^{j}\left(t, P_{k}^{j}\right)+\left(\bar{C}^{j}\right)^{\prime}\left(K^{j}\right)^{\prime} R K^{j} \bar{C}^{j}=0
$$

with the maps $\Delta^{j}$ and $\Gamma^{j}$ defined by

$$
\begin{aligned}
& \Delta^{j}, \Gamma^{j}:\left[t_{j-1}, t_{j}\right) \times H_{(n+m)(j+1)} \rightarrow H_{(n+m)(j+1)} \\
& {\left[\Delta^{j}(t, M)\right]_{i k} }=\left\{\begin{array}{ll}
\operatorname{tr}\left[D_{i}^{j}(t)^{\prime} M D j(t)\right] ; \\
0 & \text { otherwise }
\end{array} \quad i, k=1, \cdots, n\right. \\
& {\left[\Gamma^{j}(t, M)\right]_{i k} }=\left\{\begin{array}{ll}
\operatorname{tr}\left[G_{j}^{j}(t)^{\prime} M G_{k}^{j}(t)\right] ; \\
0 & \text { otherwise. }
\end{array} \quad i, k=1, \cdots, n\right.
\end{aligned}
$$

The final conditions are

$$
P_{K}^{N}(t)=Q_{f}^{N}
$$

and for $j=1, \cdots, N-1$,

$$
\left(z_{j}, \cdots, z_{0}\right) P k\left(t_{j}\right)\left(\begin{array}{c}
z_{j} \\
\vdots \\
z_{0}
\end{array}\right)=\left(z_{j} z_{j} \cdots z_{0}\right) P K^{+1}\left(t_{j}\right)\left(\begin{array}{c}
z_{j} \\
z_{j} \\
\vdots \\
z_{0}
\end{array}\right) .
$$

The last condition, after simple calculation, yields

$$
\begin{aligned}
{\left[P_{K}\left(t_{j}\right)\right]_{11} } & =\sum_{k=1}^{2} \sum_{l=1}^{2}\left[P_{k}^{j+1}\left(t_{j}\right)\right]_{k l} \\
{\left[P_{K}^{j}\left(t_{j}\right)\right]_{l 1} } & =\left[P_{K}^{j}\left(t_{j}\right)\right]_{1}=\sum_{k=1}^{2}\left[P_{K}^{j+1}\left(t_{j}\right)\right]_{k, l+1} \\
l & =2, \cdots,(n+m)(j+1) \\
{\left[P_{k}\left(t_{j}\right)\right]_{k j} } & =\left[P_{k}\left(t_{j}\right)\right]_{l k}=\left[P_{k}^{+1}\left(t_{j}\right)\right]_{k+1, l+1} \\
k, l & =2, \cdots,(n+m)(j+1)
\end{aligned}
$$

For $p k(t)$, we have

$$
\begin{aligned}
\dot{p}_{k}^{j}(t) & =-\left[\operatorname{tr}\left\{E^{j}\left(E^{j}\right)^{\prime} P_{k}^{j}(t)\right\}+\operatorname{tr}\left\{F^{j}\left(F^{j}\right)^{\prime} P_{K}(t)\right\}\right] . \\
p_{k}^{j}\left(t_{j}\right) & =0 .
\end{aligned}
$$

Finally, we have

$$
E\left(W_{k}^{j}+J_{k}^{+1}\right)=\operatorname{tr}\left[P_{k}\left(t_{j-1}\right) E\left\{Z_{i_{j-1}}^{j}\left(Z_{i_{j-1}}^{j}\right)^{\prime}\right]+p k\left(t_{j-1}\right)\right.
$$

The determination of the optimal sequence of controls $U_{1 z}^{*}, \cdots, U_{N i}^{*}$ can be accomplished in two stages.

1) First, find $K^{N}(t)$ for which $J_{K}^{N}$ given by (3.17) is a minimum, where $P_{K}^{N}(t)$ and $p_{K}^{N}(t)$ are solutions of (3.12), (3.14), and (3.16). Denote the optimal $P_{K}^{N}(t)$ and $p_{K}^{N}(t)$ by $P_{K}^{N *}(t)$ and $p_{K}^{N^{*}}(t)$.

2) Suppose that optimal $K^{n *}(t), n=j+1, \cdots, N$, has been determined. Determine $K^{j}(t)$ for which $E\left(W_{k}^{j}+J_{K}^{(j+1) *}\right)$ given by (3.17) is a minimum, where $P \dot{k}(t)$ and $p \dot{k}(t)$ are solutions of (3.12), (3.15a)-(3.15c), and (3.16), where in the right-hand sides of $(3.15 \mathrm{a})-(3.15 \mathrm{c})$ we use $P_{K}(j+1) *\left(t_{j}\right)$, the optimal values of $P_{K}^{(j+1)}\left(t_{j}\right)$. This is possible because $E\left\{Z_{t_{j-1}}^{j}\left(Z_{t_{j-1}}^{j}\right)^{\prime}\right\}$ is independent of the choice of $K^{j}(t)$ in $\left[t_{j-1}, t_{j}\right)$.

The minimizations may be carried out successively. We briefly outline the minimization procedure for 1 ). The rest can be performed similarly. We may write

$$
J_{K}^{N}=\operatorname{tr}\left[P_{K}^{N}\left(t_{j-1}\right) E\left\{Z_{t_{N-1}^{N}}^{N}\left(Z_{t_{N-1}}^{N}\right)^{\prime}\right\}+\int_{T}^{t_{N-1}} \dot{p}_{K}^{N}(s) d s .\right.
$$

Take $P_{\mathcal{K}}^{N}(t), t \in\left[t_{N-1}, T\right)$ as the "state matrix" and $K^{N}(t)$ as the "control matrix" and define the Hamiltonian

$$
H\left(P_{K}^{N}(t), S_{K}^{N}(t), t, K^{N}(t)\right) \triangleq \dot{p}_{K}^{N}(t)+\operatorname{tr}\left(\dot{P}_{K}^{N}(t) S_{K}^{N}(t)\right)
$$

with $S_{K}^{N}(t)$ denoting the "costate matrix." The matrix maximum principle gives the following result (see [2] for details).

Assuming that the matrix $\bar{C}^{N} S_{K}^{N}\left(\bar{C}^{N}\right)^{\prime}$ is invertible for $t \in\left[t_{N-1}, T\right]$ there is a unique $K^{N^{*}}(t)$ that minimizes $J_{K}^{N}$, given by

$$
K^{N *}(t)=R(t)^{-1} B^{N}(t)^{\prime} P_{K}^{N *}(t)\left(\bar{C}^{N}\right)\left(\bar{C}^{N}\right)^{\prime}\left(\bar{C}^{N} S_{K}^{N *}\left(\bar{C}^{N}\right)^{\prime}\right)^{-t}
$$

where $S_{K}^{N *}(t)$ is the unique solution of

$$
\begin{aligned}
\dot{S}_{K}^{N *}= & S_{K}^{N *}\left\{A^{N}-B^{N} K^{N *} \bar{C}^{N}\right\}+\left\{A^{N}-B^{N} K^{N *} \bar{C}^{N}\right\} S_{K}^{N *} \\
& +M\left(t, S_{K}^{N *}\right)+N\left(t, S_{K}^{N *}\right)+E^{N}\left(E^{N}\right)^{\prime}+F^{N}\left(F^{N}\right)^{\prime} \\
S_{K}^{N *}\left(t_{N-1}\right)= & E\left\{Z_{t_{N-1}^{N *}}^{N *}\left(Z_{t_{N-1}}^{N *}\right)^{\prime}\right\}
\end{aligned}
$$

where $M:[0, T] \times H_{n+m} \rightarrow H_{n+m}$ with

$$
M(t, S)=\sum_{i, j=1}^{n} S_{i j} D_{i} .
$$

We see that the optimal controls $K^{j *}(t)$ need solutions of nonlinear two-point boundary value problems, $j=N, \cdots, 1$.

\section{CONCLUSION}

We indicated a solution technique for obtaining the optimal linear stochastic control problem for dynamical systems with multiplicative noise and with quadratic criterion, where observations are available only at discrete-time points. The details may be found in [5]. 


\section{REFERENCES}

[1] P. J. Mclane, "Linear optimal stochastic control using instantaneous output feedback," Int. J. Contr, vol. 13, pp. 383-396, 1971.

[2] M. Alhans, "The matrix minimum principle," Inform. Contr., vol. 11, pp. 592-606, 1968.

[3] A. Friedman, Partial Differential Equations of Parabolic Type. Englewood Cliffs, NJ: Prentice-Hall, 1964.

[4] A. V. Skorokhod, Studies in the Theory of Random Processes. Reading, MA Addison-Wesley, 1965 .

[5] A. Bagchi and T. Schilperoort, "Optimal linear stochastic control for systems with multiplicative noise," Dep. Appl. Math., Twente Univ. Technol, Enschede, The Netherlands, Memo. 252, Mar. 1979.

\section{Suboptimal Control Using Pade Approximation Techniques}

\section{JAYANTA PAL}

Abstract-A method is given for the design of suboptimal controllers for single-input single-output systems using partial state feedback. This is based on the Pade approximation technique for model order reduction.

\section{INTRODUCTION}

One of the drawbacks of optimal control theory is that it requires feedback from all the state variables that are defined to describe the dynamics of the plant. Unfortunately, the whole state vector is seldom available for measurement. One alternative is to reconstruct the missing states by using a Kalman filter or an observer. This introduces high-order dynamics in the control function and leads to a complicated and costly controller. This has motivated the design of incomplete state feedback suboptimal control laws using only the measurable states [1], [2].

In this paper a method for suboptimal controller design using measurable states for feedback is proposed. The suboptimal controller is derived from the "optimal" one by introducing constraints in the control structure. The Pade approximation technique for model order reduction [3] is used for arriving at the controller parameters.

\section{Thi Design Method}

The basic optimal control problem may be stated as follows. Consider the $\boldsymbol{n}$ th order single-input single-output linear dynamic system described by

$$
\begin{aligned}
& \dot{x}=A x(t)+b u(t) \\
& y=c^{T} x(t)
\end{aligned}
$$

with the quadratic cost function

$$
J=\int_{0}^{\infty}\left\{x^{T}(t) Q x(t)+r u^{2}(t)\right\} d t
$$

where $Q$ is an $(n \times n)$ positive semidefinite matrix and $r$ is a positive weight. $A, b$, and $c$ are matrices of appropriate dimensions. It is well known that the optimal feedback control law is a linear combination of the state variables

$$
u(t)=-r^{-1} b^{T} P x=-k^{T} x
$$

where $P$ is a symmetric positive definite matrix whose elements may be found by solving the matrix Riccati equation

$$
A^{T} P+P A-P b r^{-1} b^{T} P+Q=0 .
$$

Manuscript received June 29, 1979; revised April 9, 1980.

The author is with the Department of Electrical Engineering, University of Roorkee, Roorkee, India.
The closed-loop transfer function with the optimal controller of (3) is

$$
\begin{aligned}
T^{*}(s) & =c^{T}\left[s I-A+b k^{T}\right]^{-1} b \\
& =\frac{b_{0}+b_{1} s+b_{2} s^{2}+\cdots+b_{m-1} s^{m-1}+b_{m} s^{m}}{a_{0}+a_{1} s+a_{2} s^{2}+\cdots+a_{n-1} s^{n-1}+a_{n} s^{n}} \\
& =d_{0}+d_{1} s+d_{2} s^{2}+\cdots
\end{aligned}
$$

where (6) is the power series expansion of (5) about $s=0$. Restricting the admissible control law to be linear and utilizing only the available states for feedback, the suboptimal controller may be specified as

$$
\tilde{u}=-\tilde{k}^{T} x
$$

where $\tilde{k}_{j}=0$ if $x_{j}(t)$ is not available for feedback. Assuming that such a suboptimal controller exists, i.e., system (1) may be stabilized by the control law (7), the overall transfer function becomes

$$
\begin{aligned}
\tilde{T}(s) & =c^{T}\left[s I-A+b \tilde{k}^{T}\right]^{-1} b \\
& =\frac{b_{0}+b_{1} s+b_{2} s^{2}+\cdots+b_{m-1} s^{m-1}+b_{m} s^{m}}{f_{0}+f_{1} s+f_{2} s^{2}+\cdots+f_{n-1} s^{n-1}+f_{n} s^{n}} .
\end{aligned}
$$

For the choice of control laws in (3) and (7), the numerator polynomials in (5) and (8) will be the same. $f_{j}(j=0,1, \cdots, n)$ will contain the unknown feedback parameters in $\tilde{k}^{T}$. The incomplete state feedback problem is concerned with finding the elements of $\tilde{k}^{T}$ on some basis. For the suboptimal system response to be favorably comparable with that of the optimal one, the function in (8) should approximate $T^{*}(s)$ in $(5)$ in some sense. The design technique is to use the Pade approximation method to find the unknown controller parameters in $\tilde{k}^{T}$.

For $\tilde{T}(s)$ to approximate $T^{*}(s)$ in the Pade sense, we have [3]

$$
\begin{aligned}
b_{0} & =f_{0} d_{0} \\
b_{1} & =f_{0} d_{1}+f_{1} d_{0} \\
b_{2} & =f_{0} d_{2}+f_{1} d_{1}+f_{2} d_{0} \\
\vdots & \cdots \cdots \cdots \cdots \cdots \cdots \\
b_{m} & =f_{0} d_{m}+f_{1} d_{m-1}+f_{2} d_{m-2}+\cdots+f_{m} d_{0} \\
0 & =f_{0} d_{m+1}+f_{1} d_{m}+f_{2} d_{m-1}+\cdots+f_{m+1} d_{0} \\
\cdots & \cdots \cdots \cdots \cdots \cdots \cdots \cdots \cdots \cdots \cdots \cdots+f_{n-1} d_{m+1}+f_{n} d_{m} .
\end{aligned}
$$

Assuming that $v$ state variables $(v<n)$ are available for feedback, the $v$ unknown elements of $\bar{k}^{T}$ can be explicitly determined by solving the first $v$ linear equations in (9). The method is illustrated by the following example.

\section{EXAMPLE}

The voltage regulator example [4] is given by (1), where

$$
\begin{aligned}
& A=\left[\begin{array}{rrccr}
-0.2 & 0.5 & 0.0 & 0.0 & 0.0 \\
0.0 & -0.5 & 1.6 & 0.0 & 0.0 \\
0.0 & 0.0 & -14.29 & 85.715 & 0.0 \\
0.0 & 0.0 & 0.0 & -25.0 & 75.0 \\
0.0 & 0.0 & 0.0 & 0.0 & -10.0
\end{array}\right] \\
& b=\left[\begin{array}{lllll}
0.0 & 0.0 & 0.0 & 0.0 & 30.0
\end{array}\right]^{T} .
\end{aligned}
$$

Choosing $Q=\operatorname{diag}\{1,0,0,0,0\}$; and $r=1$, in (2), on solving (4) we get

$$
k^{T}=-\left[\begin{array}{lllll}
0.9245 & 0.1711 & 0.0161 & 0.0492 & 0.2643
\end{array}\right] \text {. }
$$

Using the above $k^{T}$, we have $T^{*}(s)=432.0 / a(s)$, where

$a(s)=432.05518+169.89431 s+33.364525 s^{2}$

$$
+3.3945708 s^{3}+0.1621612 s^{4}+0.0028 s^{5} \text {. }
$$

